PROCEEDINGS OF THE

AMERICAN MATHEMATICAL SOCIETY

Volume 138, Number 2, February 2010, Pages 655-661

S 0002-9939(09)10092-8

Article electronically published on October 6, 2009

\title{
REMOVABLE SETS FOR THE FLUX OF CONTINUOUS VECTOR FIELDS
}

\author{
SÉBASTIEN DE VALERIOLA AND LAURENT MOONENS
}

(Communicated by Tatiana Toro)

\begin{abstract}
We show that any closed set $E$ having a $\sigma$-finite $(n-1)$-dimensional Hausdorff measure does not support the nonzero distributional divergence of a continuous vector field; in particular it has the property that any $C^{1}$ function in $\mathbb{R}^{n}$ that is harmonic outside it is harmonic in $\mathbb{R}^{n}$. We also exhibit a compact set $E$ having Hausdorff dimension $n-1$, supporting the nonzero distributional divergence of a continuous vector field yet having the property that any $C^{1}$ function that is harmonic outside $E$ is harmonic in $\mathbb{R}^{n}$.
\end{abstract}

\section{INTRODUCTION}

The problem of removing singularities for the equation $\operatorname{div} v=0$ can be stated as follows: given a collection $\mathcal{B}$ of locally bounded, measurable vector fields in $\mathbb{R}^{n}$, give conditions on a compact set $E$ guaranteeing that each vector field $v \in \mathcal{B}$ satisfying $\operatorname{div} v=0$ outside $E$ (in the distributional sense) satisfies $\operatorname{div} v=0$ in $\mathbb{R}^{n}$ (in the distributional sense, too).

This problem has been studied for various classes of functions. When $\mathcal{B}$ is the collection of all (locally) bounded, measurable vector fields, the compact removable sets are exactly those having zero $(n-1)$-dimensional Hausdorff measure (see [2], [8] and [1]). In this short paper, we focus on the case when $\mathcal{B}$ is the collection of all continuous vector fields in $\mathbb{R}^{n}$. Theorem 5.2 (second part) in [1] states that removable sets in this context have Hausdorff dimension at most $n-1$. On the other hand, it is a rather easy observation (see [9, Lemma 8.18] for example) that any compact set $E$ satisfying $\mathcal{H}^{n-1}(E)<\infty$ is removable. In this paper, we extend this property to closed sets having $\sigma$-finite $(n-1)$-dimensional Hausdorff measure (Theorem[12). In particular, each function $u$ that is $C^{1}$ in $\mathbb{R}^{n}$ and harmonic outside a closed set having $\sigma$-finite $(n-1)$-dimensional Hausdorff measure is harmonic in $\mathbb{R}^{n}$ (apply the removability property to the vector field $v=\nabla u$ ).

We then show that the dimension condition does not characterize removable sets. In particular, we exhibit (Theorem [13) a compact set $E$ that has Hausdorff dimension $n-1$, is not removable, yet satisfies the following condition: any $C^{1}$ function $u: \mathbb{R}^{n} \rightarrow \mathbb{R}$ harmonic outside $E$ is harmonic in $\mathbb{R}^{n}$.

Received by the editors January 7, 2009, and, in revised form, June 10, 2009.

2000 Mathematics Subject Classification. Primary 49Q15; Secondary 35B60.

The second author is an aspirant of the Fonds de la Recherche scientifique — FNRS (Belgium).

(C)2009 American Mathematical Society Reverts to public domain 28 years from publication 


\section{Preliminaries AND NOtation}

In the sequel, we work in Euclidean space $\mathbb{R}^{n}, n \geqslant 1$. The Euclidean norm of $x=\left(x_{1}, \ldots, x_{n}\right) \in \mathbb{R}^{n}$ is denoted $|x|$; for $r>0$ let $B[x, r]=\left\{y \in \mathbb{R}^{n}:|x-y| \leqslant r\right\}$ and $Q[x, r]=\left\{y \in \mathbb{R}^{n}:\left|y_{i}-x_{i}\right| \leqslant r, 1 \leqslant i \leqslant n\right\}$. We write $B[r]$ and $Q[r]$ instead of $B[0, r]$ and $Q[0, r]$, respectively. A cube is a set of the form $Q[x, r]$ for $x \in \mathbb{R}^{n}$ and $r>0$.

Given $E \subseteq \mathbb{R}^{n}$, we will write $E \subset \subset U$ to indicate that the closure of $E$ is a compact subset of the open set $U \subseteq \mathbb{R}^{n}$. We further denote by $\mathcal{L}^{n}(E)$ or $|E|$ the Lebesgue (outer) measure of $E$; in particular we define $\boldsymbol{\alpha}(n):=|B[1]|$. The notation $d(E)$ stands for the diameter of $E$, and for $0 \leqslant s \leqslant n$ we let $\mathcal{H}^{s}(E)$ (resp. $\mathcal{S}^{s}(E)$ ) denote the (normalized by $\boldsymbol{\alpha}(s) 2^{-s}$ ) $s$-dimensional Hausdoff measure (resp. the $s$-dimensional spherical Hausdorff measure) of $E$. One has $\mathcal{H}^{s}(E) \leqslant \mathcal{S}^{s}(E) \leqslant$ $2^{s} \mathcal{H}^{s}(E)$, as is easily checked. We also say that $E$ is thin in case $\mathcal{H}^{n-1} L E$ is $\sigma$-finite.

Given $f: X \rightarrow Y$ and $A \subseteq X$, we let $f \uparrow A$ denote the restriction of $f$ to $A$. The restriction of an outer measure $\mu$ on $\mathbb{R}^{n}$ to $E \subseteq \mathbb{R}^{n}$ is the outer measure $\mu\llcorner E$ defined by $\mu\llcorner E(B):=\mu(B \cap E)$.

We denote by $\|f\|_{\infty}$ and $\|f\|_{1}$ the $L^{\infty}$ - and $L^{1}$-norms of $f: \mathbb{R}^{n} \rightarrow \mathbb{R}^{m}$, respectively. Given $f \in L^{1}\left(\mathbb{R}^{n}\right)$, we denote by $\|D g\|$ its total variation. For $0 \leqslant k \leqslant \infty$, we give $C^{k}\left(\mathbb{R}^{n}, \mathbb{R}^{m}\right), C_{c}^{k}\left(\mathbb{R}^{n}, \mathbb{R}^{m}\right)$ and $C^{k}\left(\mathbb{R}^{n}\right), C_{c}^{k}\left(\mathbb{R}^{n}\right)$ their usual meanings.

\section{Charges And fluXes}

The flux of any (locally integrable) $v: \mathbb{R}^{n} \rightarrow \mathbb{R}^{n}$ is the distribution $F_{v}$ : $C_{c}^{\infty}\left(\mathbb{R}^{n}\right) \rightarrow \mathbb{R}$ defined by $F_{v}(\varphi)=-\int_{\mathbb{R}^{n}} v(x) \cdot \nabla \varphi(x) d x$. We observe an interesting continuity property of the flux of a continuous vector field.

Proposition 1. Given $v \in C^{0}\left(\mathbb{R}^{n}, \mathbb{R}^{n}\right), \varepsilon>0, r>0$ and $C>0$, there exists $\delta>0$ such that $F_{v}(\varphi) \leqslant \varepsilon$ provided $\varphi \in C_{c}^{\infty}\left(\mathbb{R}^{n}\right)$ satisfies $\{\varphi \neq 0\} \subseteq B[r],\|\nabla \varphi\|_{1} \leqslant C$ and $\|\varphi\|_{1} \leqslant \delta$.

Proof. Fix $v \in C^{0}\left(\mathbb{R}^{n}, \mathbb{R}^{n}\right), \varepsilon>0$ and choose $w \in C_{c}^{1}\left(\mathbb{R}^{n}, \mathbb{R}^{n}\right)$ satisfying $\mid v(x)-$ $w(x) \mid \leqslant \varepsilon / 2 C$ for each $x \in B[r]$. Let $\theta:=1+\|\operatorname{div} w\|_{\infty}$ and say $\delta:=\varepsilon /(2 \theta)$. For $\varphi \in C_{c}^{\infty}\left(\mathbb{R}^{n}\right)$ satisfying $\{\varphi \neq 0\} \subseteq B[r],\|\nabla \varphi\|_{1} \leqslant C$ and $\|\varphi\|_{1} \leqslant \delta$, compute using the integration by parts formula:

$$
\begin{aligned}
F_{v}(\varphi)=\int_{\mathbb{R}^{n}}[v(x)-w(x)] \cdot \nabla \varphi(x) d x+\int_{\mathbb{R}^{n}} \varphi(x) \operatorname{div} w(x) d x & \\
& \leqslant \frac{\varepsilon}{2 C}\|\nabla \varphi\|_{1}+\theta\|\varphi\|_{1} \leqslant \varepsilon .
\end{aligned}
$$

The proof is complete.

Remark 2. According to the approximation property of $B V$ functions (see [5, Section 5.2.2]), it is obvious that given $v \in C^{0}\left(\mathbb{R}^{n}, \mathbb{R}^{n}\right), F_{v}$ extends to the linear functional

$$
F_{v}: B V_{c}\left(\mathbb{R}^{n}\right) \rightarrow \mathbb{R} ; g \mapsto-\int_{\mathbb{R}^{n}} v \cdot d[D g]
$$

on the space $B V_{c}\left(\mathbb{R}^{n}\right)$ of compactly supported functions having bounded variation in $\mathbb{R}^{n}$. Furthermore for any $\varepsilon>0, r>0$ and $C>0$ there exists $\delta>0$ such that $F(g) \leqslant \varepsilon$ provided $g \in B V_{c}\left(\mathbb{R}^{n}\right)$ satisfies $\{g \neq 0\} \subseteq B[r],\|D g\| \leqslant C$ and $\|g\|_{1} \leqslant \delta$. 
Given $r>0$ and $C>0$, let

$$
B V_{r, C}:=\left\{g \in B V_{c}\left(\mathbb{R}^{n}\right): \operatorname{supp} g \subseteq B[r],\|D g\| \leqslant C\right\}
$$

and denote by $\mathfrak{B}$ the collection of all absorbing, balanced convex sets $\mathbf{U} \subseteq B V_{c}\left(\mathbb{R}^{n}\right)$ having the property that for all $r>0$ and $C>0$, the set $\mathbf{U} \cap B V_{r, C}$ is a relative $L^{1}$-neigborhood of $g$ in $B V_{r, C}$. According to [3, Theorem 3.3], for example, $\mathfrak{B}$ is a neighborhood basis at 0 for a locally convex topology $\mathfrak{T}$ on $B V_{c}\left(\mathbb{R}^{n}\right)$ which is characterized by the following two properties:

(A) $\mathfrak{T}$ is weaker than the $L^{1}$ topology on $B V_{r, C}$ for all $r>0, C>0$;

(B) for any locally convex space $X$, a linear map $f: B V_{c}\left(\mathbb{R}^{n}\right) \rightarrow X$ is $\mathfrak{T}$ continuous whenever the restriction $f \uparrow B V_{r, C}$ is $L^{1}$-continuous on $B V_{r, C}$ for all $r>0, C>0$.

Given $v \in C^{0}\left(\mathbb{R}^{n}, \mathbb{R}^{n}\right)$, condition (B) together with Remark 2 implies in particular that $F_{v}$ is $\mathfrak{T}$-continuous on $B V_{c}\left(\mathbb{R}^{n}\right)$.

Definition 3. A $\mathfrak{T}$-continuous linear functional $F$ on $B V_{c}\left(\mathbb{R}^{n}\right)$ is called a charge.

The following theorem, due to T. De Pauw and W.F. Pfeffer [4], is very deep and identifies fluxes and charges.

Theorem 4 (De Pauw-Pfeffer). Given a charge $F$, there exists a vector field $v \in$ $C^{0}\left(\mathbb{R}^{n}, \mathbb{R}^{n}\right)$ for which $F=F_{v}$.

On the other hand, N.C. Phuc and M. Torres [11 give a sufficient condition for a Radon measure to define a charge.

Theorem 5. Assume that $\mu$ is a Radon measure in $\mathbb{R}^{n}$ and that for each compact set $K \subseteq \mathbb{R}^{n}$ one has

$$
\lim _{\delta \rightarrow 0} \sup _{0<r<\delta} \sup _{x \in K} \frac{\mu(B[x, r])}{r^{n-1}}=0 .
$$

Then the linear functional

$$
C_{c}^{\infty}\left(\mathbb{R}^{n}\right) \rightarrow \mathbb{R}, \varphi \mapsto \int_{\mathbb{R}^{n}} \varphi d \mu
$$

extends in a unique way to a charge.

\section{REMOVING THIN SETS}

The following lemma will be useful in the sequel.

Lemma 6. Assume that $E \subseteq \mathbb{R}^{n}$ is bounded and satisfies $\mathcal{H}^{n-1}(E)<\infty$. For all $\varepsilon>0$ and $\eta>0$, there exists an open set $U$ containing $E$ and satisfying the following conditions:

(A) $|U| \leqslant \varepsilon$

(B) $\left\|D \chi_{U}\right\| \leqslant \eta+\boldsymbol{\beta}(n) \mathcal{H}^{n-1}(E)$,

where $\boldsymbol{\beta}(n):=2^{n-1}$.

Proof. First, observe that the dominated convergence theorem yields $|B(E, r)| \leqslant \varepsilon$ in case $r>0$ is sufficiently small, say $r \leqslant \delta$. Continue by choosing a countable family $\left(B_{k}\right)_{k \geqslant 1}$ of open balls with diameter less than $\delta$ such that $U:=\bigcup_{k=1}^{\infty} B_{k} \supseteq E$ and $2^{1-n} \boldsymbol{\alpha}(n-1) \sum_{k=1}^{\infty} d\left(B_{k}\right)^{n-1} \leqslant \mathcal{S}^{n-1}(E)+\eta$. Without loss of generality, we 
can assume that $B_{k} \cap E \neq \emptyset$ for each $k \geqslant 1$; in particular we have $U \subseteq B(E, \delta)$ and hence $|U| \leqslant \varepsilon$. On the other hand,

$$
\left\|D \chi_{U}\right\| \leqslant \sum_{k=1}^{\infty}\left\|D \chi_{B_{k}}\right\|=2^{1-n} \boldsymbol{\alpha}(n-1) \sum_{k=1}^{\infty} d\left(B_{k}\right)^{n-1} \leqslant 2^{n-1} \mathcal{H}^{n-1}(E)+\eta,
$$

and the proof is complete.

Lemma 7. If $E \subseteq \mathbb{R}^{n}$ is closed and thin, then the collection of all $g \in B V_{c}\left(\mathbb{R}^{n}\right)$ that vanishes in a neighborhood of $E$ is $\mathfrak{T}$-dense in $B V_{c}\left(\mathbb{R}^{n}\right)$.

Proof. First, write $E$ as an increasing union $\bigcup_{k=1}^{\infty} E_{k}$ where $\mathcal{H}^{n-1}\left(E_{k}\right)<\infty$ for each $k \geqslant 1$. Replacing if necessary $E_{k}$ by $E_{k} \cap B(k)$ we may assume that $E_{k}$ is bounded for each $k \geqslant 1$. We further define for each $k \geqslant 1$ a constant $C_{k}:=$ $\|g\|_{\infty}\left[1+\sum_{j=1}^{k} \mathcal{H}^{n-1}\left(E_{j}\right)\right]+\|D g\|$ and choose $r>0$ so that $\operatorname{supp} g \subseteq B[r]$.

Now fix $\mathbf{U}$ a $\mathfrak{T}$-neighborhood of 0 in $B V_{c}\left(\mathbb{R}^{n}\right)$. According to the definition of $\mathfrak{T}$, there exists $\delta_{k}>0$ such that any $h \in B V_{c}\left(\mathbb{R}^{n}\right)$ satisfying supp $h \subset B[r],\|D h\| \leqslant C_{k}$ and $\|h\|_{1} \leqslant \delta_{k}$ belongs to $2^{-k} \mathbf{U}$.

For each $k \geqslant 1$, choose according to Lemma [6 an open set $U_{k} \supseteq E_{k} \cap \operatorname{supp} g$ satisfying $\|g\|_{\infty}\left|U_{k}\right| \leqslant \delta_{k}$ and $\left\|D \chi_{U_{k}}\right\| \leqslant 2^{-k}+\boldsymbol{\beta}(n) \mathcal{H}^{n-1}\left(E_{k}\right)$. Observe that by compactness, there exists $l \geqslant 1$ such that $E \cap \operatorname{supp}(g) \subseteq \bigcup_{k=1}^{l} U_{k}$.

Define $g_{1}:=g \chi_{U_{1}}$, and for $k \geqslant 2$, let $g_{k}:=g \chi_{U_{k} \backslash \bigcup_{j=1}^{k-1} U_{j}}$. Fix $k \geqslant 1$. We obviously have supp $g_{k} \subseteq B[r]$ and $\left\|g_{k}\right\|_{1} \leqslant \delta_{k}$, while

$\left\|D g_{k}\right\| \leqslant\|g\|_{\infty} \sum_{j=1}^{k}\left\|D \chi_{U_{j}}\right\|+\|D g\| \leqslant\|g\|_{\infty}\left[1+\boldsymbol{\beta}(n) \sum_{j=1}^{k} \mathcal{H}^{n-1}\left(E_{j}\right)\right]+\|D g\| \leqslant C_{k}$,

where we used [10, Proposition 1.8.4] for example to estimate $\left\|D \chi_{U_{k} \backslash \bigcup_{j=1}^{k-1} U_{j}}\right\|$. Consequently we get $g_{k} \in 2^{-k} \mathbf{U}$.

We finish the argument by observing that $g^{\prime}:=\sum_{k=1}^{l} g_{k}$ equals $g$ on a neighborhood of $E \cap \operatorname{supp} g$ (and hence equals $g$ on a neighborhood of $E$ ) and belongs to $\sum_{k=1}^{l} 2^{-k} \mathbf{U} \subseteq \mathbf{U}$.

Observation 8. It is not sufficient that $E$ be thin for the conclusion of Lemma 7 to hold, as the following example shows.

Example 9. Assume that $n=1$ and let $E$ denote the set of rational numbers (which is thin but not closed). If $g \in B V_{c}(\mathbb{R})$ vanishes on an open set $U \supset E$, then choose according to [1, Theorem 3.27] a function $\tilde{g}=g$ a.e. whose pointwise variation equals $\|D g\|$. We claim that $\{\tilde{g} \neq 0\}$ is countable, for otherwise given any $k \geqslant 1$, one would be able to find a finite set of $B_{k} \subset\{\tilde{g} \neq 0\}$ such that $\sum_{x \in E_{k}} \tilde{g}(x) \geqslant k$. This would imply that the pointwise variation of $\tilde{g}$ is infinite because $\{\tilde{g}=0\}$ is dense in $\mathbb{R}$. Consequently $g=0$ a.e.

Definition 10. The support of a charge $F$, denoted $\operatorname{supp} F$, is the complementary set of the largest open set $U \subseteq \mathbb{R}^{n}$ having the following property: $F(g)=0$ provided $g \in B V_{c}\left(\mathbb{R}^{n}\right)$ satisfies $\{g \neq 0\} \subset \subset U$.

Observation 11. Given a charge $F$, the support of $F$ coincides with the support (defined in the usual way) of the distribution $F\left\lceil C_{c}^{\infty}\left(\mathbb{R}^{n}\right)\right.$ uniquely determining $F$.

An immediate corollary of Lemma 7 is the following.

Theorem 12. If $F$ is a charge and if $\operatorname{supp} F$ is thin, then $F \equiv 0$. 


\section{A nonRemovable Set With Dimension $n-1$}

Given a cube $Q$, we denote by $\sigma(Q)$ the length of its edges. For $0<\lambda<1 / 2$, we let $\mathcal{E}(Q, \lambda)$ stand for the collection of $2^{n}$ cubes contained in $Q$ whose edges have length $\lambda \sigma(Q)$, arranged in such a way that each cube of $\mathcal{E}(Q, \lambda)$ has a common vertex with $Q$.

Fix a sequence $\lambda=\left(\lambda_{k}\right)_{k \geqslant 1} \subseteq(0,1 / 2)$. Write $E_{0}:=[0,1]^{n}$ and $\varepsilon_{0}=\left\{E_{0}\right\}$. For each $k \geqslant 1$, define inductively

$$
\varepsilon_{k}\left(\lambda_{1}, \ldots, \lambda_{k}\right):=\bigcup\left\{\mathcal{E}\left(Q, \lambda_{k}\right): Q \in \mathcal{E}_{k-1}\left(\lambda_{1}, \ldots, \lambda_{k-1}\right\}\right.
$$

and $E_{k}\left(\lambda_{1}, \ldots, \lambda_{k}\right):=\bigcup \mathcal{E}_{k}\left(\lambda_{1}, \ldots, \lambda_{k}\right)$ (or briefly $\mathcal{E}_{k}$ and $E_{k}$ when the underlying sequence is clear). In particular, each cube in $\mathcal{E}_{k}$ has edges of length $\sigma_{k}:=\lambda_{1} \ldots \lambda_{k}$. Finally we let $E(\lambda)=\bigcap_{k=1}^{\infty} E_{k}$.

Associated to $\lambda$, we choose $h:[0, \infty) \rightarrow[0, \infty)$ as a nondecreasing function satisfying $h(0)=0$ and $h\left(\sigma_{k}\right)=2^{-k n}$ for each $k \geqslant 1$. Recall that the Hausdorff $h$-measure of $E(\lambda)$ is positive and finite (see [7, Section 4.9]).

Theorem 13. There is a compact set $E \subseteq \mathbb{R}^{n}$ having Hausdorff dimension $n-1$ and satisfying the following properties:

(A) there exists $v \in C^{0}\left(\mathbb{R}^{n}, \mathbb{R}^{n}\right)$ and a nonzero Radon measure $\mu$ supported in $E$ such that $\operatorname{div} v=\mu$ (in the distribution sense);

(B) if $u \in C^{1}\left(\mathbb{R}^{n}\right)$ is harmonic outside $E$, then $u$ is harmonic in $\mathbb{R}^{n}$.

In particular, $\mathcal{H}^{n-1}\llcorner E$ is not $\sigma$-finite.

Proof. For $k \geqslant 3$, let $\ell_{k}=2^{-\frac{n}{n-1}} \log k / \log (k-1)$ and assume that $\lambda_{k}=\ell_{k}$ for $k$ sufficiently large. Then:

(1) $\mathcal{H}^{n-1}\llcorner E(\lambda)$ is not $\sigma$-finite,

(2) $\operatorname{dim}_{\mathcal{H}}(E(\lambda))=n-1$;

the proofs of both facts are standard.

Given $k \geqslant 1$, we associate to $E_{k}$ a Radon measure $\mu_{k}:=\left|E_{k}\right|^{-1} \mathcal{L}^{n} L E_{k}$ supported in $E_{k}$. Observe that $\mu_{k}\left(\mathbb{R}^{n}\right)=1$ for each $k$. Hence, [7, Theorem 1.23] ensures that there exists a subsequence of $\left(\mu_{k}\right)$ weakly*-converging to a Radon measure $\mu$ supported in $E(\lambda)$.

We now turn to showing that

$$
\lim _{\delta \rightarrow 0} \sup _{0<r<\delta} \sup _{x \in \mathbb{R}^{n}} \frac{\mu(B[x, r])}{r^{n-1}}=0 .
$$

Observe that it suffices to show (1) with $\mathbb{R}^{n}$ replaced by $E(\lambda)$ and $B[x, r]$ replaced by $Q[x, r]$.

Claim 14. Fix $x \in E(\lambda)$ and $k$ sufficiently large for $\lambda_{k}=\ell_{k}$ to hold. If $\sigma_{k+1} \leqslant r \leqslant$ $\sigma_{k}$, then

$$
\frac{\mu(Q[x, r])}{r^{n-1}} \leqslant 2^{-k n}
$$

Proof of the claim. Observe that $Q[x, r]$ is contained in $2^{n}$ cubes $K_{1}, \ldots, K_{2^{n}}$ having $x$ as a common vertex and edges of length $\sigma_{k}$. In particular, we get for $1 \leqslant i \leqslant 2^{n}$ and some arbitrary but fixed $Q_{*} \in \mathcal{E}_{k-1}$ :

$$
\left|E_{l} \cap K_{i}\right| \leqslant\left|E_{l} \cap Q\right| \text { for any } Q \in \mathcal{E}\left(Q_{*}, \lambda_{k}\right),
$$


and consequently

$$
\mu_{l}(Q[x, r]) \leqslant 2^{-l n} \sigma_{l}^{-n}\left|E_{l} \cap Q_{*}\right|=2^{-l n} 2^{(k+1) n} .
$$

This yields

$$
\frac{\mu_{l}(Q[x, r])}{r^{n-1}} \leqslant \frac{2^{(k-l+1) n}}{\sigma_{k+1}^{n-1}} \leqslant 2^{-l n} \leqslant 2^{-k n} ;
$$

the conclusion follows for $\mu(Q[x, r]) \leqslant \varlimsup_{l} \mu_{l}(Q[x, r])$.

Claim 14 together with Theorems 4 and 5 yields a vector field $v \in C^{0}\left(\mathbb{R}^{n}, \mathbb{R}^{n}\right)$ such that $\operatorname{div} v=\mu$ in the distribution sense. Moreover, the equality $\mu\left(\mathbb{R}^{n}\right)=1$ ensures that $\mu$ is nonzero. This proves property (A) of Theorem 13 .

The proof of property (B) uses a recent result by A. Ruiz de Villa and X. Tolsa 12. Observe that for $k_{0}$ sufficiently large

$$
\tau:=\sum_{k=k_{0}}^{\infty} \frac{1}{\left(2^{k n} \sigma_{k}^{n-1}\right)^{2}}=\sum_{k=k_{0}}^{\infty}\left(\frac{1}{\log k}\right)^{2(n-1)}=\infty .
$$

According to [12, Theorem 6], we get

$\kappa^{c}(E(\lambda)):=\sup \left\{|\langle\Delta u, 1\rangle|: u \in C^{1}\left(\mathbb{R}^{n}\right), \operatorname{supp}(\Delta u) \subseteq E,\|\nabla u\|_{\infty} \leqslant 1\right\}=\tau^{-1 / 2}=0$.

It follows from [6, Proposition 2.2] that the above equality is equivalent to property (B). The proof is complete.

\section{ACKNOWLEDGEMENT}

The authors want to thank the referee for suggesting the topological argument (Lemma 7) which considerably simplifies the proof of Theorem 12 .

\section{REFERENCES}

[1] Luigi Ambrosio, Nicola Fusco, and Diego Pallara. Functions of bounded variation and free discontinuity problems. Oxford Mathematical Monographs. The Clarendon Press, Oxford University Press, New York, 2000. MR.1857292 (2003a:49002)

[2] Thierry De Pauw. On the exceptional sets of the flux of a bounded vectorfield. J. Math. Pures Appl. (9), 82(9):1191-1217, 2003. MR2012808 (2004m:42027)

[3] Thierry De Pauw, Laurent Moonens, and Washek F. Pfeffer. Charges in middle dimensions. J. Math. Pures Appl., to appear (DOI 10.1016/j.matpur.2009.04.001), 2009.

[4] Thierry De Pauw and Washek F. Pfeffer. Distributions for which div $v=F$ has a continuous solution. Comm. Pure Appl. Math., 61(2):230-260, 2008. MR2368375 (2009e:46035)

[5] Lawrence C. Evans and Ronald F. Gariepy. Measure theory and fine properties of functions. Studies in Advanced Mathematics, CRC Press, Boca Raton, FL, 1992. MR1158660 (93f:28001)

[6] P. Mattila and P. V. Paramonov. On geometric properties of harmonic Lip ${ }_{1}$-capacity. Pacific J. Math., 171(2):469-491, 1995. MR1372240 (97b:31005)

[7] Pertti Mattila. Geometry of sets and measures in Euclidean spaces, Fractals and rectifiability, volume 44 of Cambridge Studies in Advanced Mathematics. Cambridge University Press, Cambridge, 1995. MR.1333890 (96h:28006)

[8] Laurent Moonens. Removable singularities for the equation div $v=0$. Real Anal. Exchange (30th Summer Symposium Conference):125-132, 2006. MR2323837(2008h:35013)

[9] Laurent Moonens. From Kurzweil-Henstock integration to charges in Euclidean spaces. Faculté des Sciences, École doctorale en Mathématique, Université catholique de Louvain. CIACO, Louvain-la-Neuve, 2008.

[10] Washek F. Pfeffer. Derivation and integration, volume 140 of Cambridge Tracts in Mathematics. Cambridge University Press, Cambridge, 2001. MR.1816996 (2001m:26018) 
[11] Nguyen Cong Phuc and Monica Torres. Characterizations of the existence and removable singularities of divergence-measure vector fields. Indiana Univ. Math. J., 57(4):1573-1597, 2008. MR2440874 (2009f:35026)

[12] Alex Ruiz de Villa and Xavier Tolsa. Characterization and semiadditivity of the $C^{1}$ harmonic capacity. Preprint (available on arXiv:0812.2421), 2008.

Département de Mathématique, Université Catholique de Louvain, Chemin du Cyclotron, 2, 1348 Louvain-la-neuve, Belgium

E-mail address: sebastien.devaleriola@uclouvain.be

Département de Mathématique, Université Catholique de Louvain, Chemin du Cyclotron, 2, 1348 Louvain-la-neuve, Belgium

E-mail address: laurent.moonens@uclouvain.be 